\title{
Small and Large Intestinal Guanylate Cyclase Activity in Children: Effect of Age and Stimulation by Escherichia coli Heat-Stable Enterotoxin
}

\author{
ALFREDO GUARINO, ${ }^{1}$ MITCHELL B. COHEN, AND RALPH A. GIANNELLA
}

Division of Digestive Diseases, University of Cincinnati College of Medicine and VA Medical Center and Division of Gastroenterology and Nutrition, Children's Hospital Research Foundation, Cincinnati, Ohio

\begin{abstract}
Heat-stable enterotoxin (ST) producing Escherichia coli are a common cause of diarrhea in infants. ST acts through the stimulation of the guanylate cyclasecGMP system. The effect of ST on the human intestine has not been investigated nor is any information available on the activity, distribution, or development of guanylate cyclase activity in the human intestine. The purpose of this study, therefore, was to characterize, these aspects of guanylate cyclase activity and to study the effect of ST on the activity and responsiveness of guanylate cyclase in the intestine of infants and children of various ages. We measured guanylate cyclase activity in 35 intestinal specimens, obtained operatively, from children aged 1 day to $16 \mathrm{yr}$. Guanylate cyclase activity was linear with protein concentration and time. Basal activity was similar in small intestine and in colon. In the small intestine, however, basal guanylate cyclase activity varied with age. It was maximal in children 1 day of age, and although somewhat variable, decreased with age thereafter. In colon, an age-related pattern was not found. $E$. coli ST stimulated guanylate cyclase activity in all specimens in a dose-related manner. In the small intestine ST-stimulation of guanylate cyclase was twice that found in colon. Furthermore, age affected the response of small intestinal guanylate cyclase to $\mathrm{ST}$. Maximal response to ST was observed in children 1 day of age and ST stimulation was significantly greater in children less than 1 yr of age than in older children. In the colon, the response of guanylate cyclase to ST did not change with age. The greater responsiveness of small intestinal guanylate cyclase to $\mathrm{ST}$ in younger children may, in part, explain the severity of diarrhea induced by ST at that age. (Pediatr Res 21: 551-555, 1987)
\end{abstract}

\section{Abbreviations}

ST, Escherichia coli heat-stable enterotoxin ED 50 , dose required for half maximal response HPLC, high-performance liquid chromatography RIA, radioimmunoassay

Received August 25, 1986; accepted January 6, 1987

Correspondence Ralph A. Giannella, M.D., Director, Division of Digestive Diseases (ML 595), University of Cincinnati Medical Center, Cincinnati, OH 45267.

Supported by VA Research Grant 539-3108-01, Public Health Service Grants Al 20261 and F32-DK07790-01, and by an AGA Supplemental Research Training Award.

'Present Address: Universita di Napoli, 2 Facolta di Medicina e Chirugia Istituto di Pediatria, Via S. Pansini 5, 80131 Napoli, Italy.
Guanylate cyclase [GTP pyrophosphate-lyase (cyclizing), EC 4.6.1.2], catalyzes the synthesis of cGMP from GTP (1). In the small intestine guanylate cyclase exists predominantly in the particulate form (2) and is located mainly in the brush border $(3,4)$. The enzymic activity is greatest in differentiated villous cells and least in proliferating crypt cells (3). Guanylate cyclase has been investigated in several tissues and animal models. Its size and properties, including sensitivity to the effects of modulators, seem to vary considerably (for a review see Ref. 1). A number of substances activate guanylate cyclase including unsaturated lipids, free radicals, and oxidants (1). However, it is unclear whether the activation of guanylate cyclase by these substances plays a physiologic role.

ST also activates guanylate cyclase and has been used to study its regulation (5-8). ST enterotoxins are a family of peptides of 18 or 19 amino acids $(9-11)$ that induce diarrhea by binding to a brush border membrane receptor (12), and subsequently activating the guanylate cyclase-cGMP system $(5-8,12)$. This results in impaired $\mathrm{NaCl}$ absorption and $\mathrm{Cl}$ secretion (13). It is well known that ST-producing Escherichia coli cause diarrhea especially in young children $(14,15)$. We have recently investigated the mechanisms of the increased response to ST demonstrated by younger animals (16). We have shown that a greater number of ST receptors are present in the jejunum of 14- and 21-day-old rats compared to adults. We suggested that this increased number of receptors could, in part, account for increased response and sensitivity to ST-induced secretion observed in these younger animals. However, we were unable to demonstrate an increase in ST-stimulated guanylate cyclase response in these immature animals (16).

Although guanylate cyclase activity has been detected in human colon (17), no further information is available on the activity, distribution, regulation, or the development of guanylate cyclase in human intestine.

Our aims were, therefore, to study guanylate cyclase in the human intestine, specifically: 1) to determine the activity and the relative distribution of guanylate cyclase activity in small and large intestine; 2) to determine whether guanylate cyclase has an age-related developmental pattern, and 3) to study the effects of E. coli ST on guanylate cyclase activity and to establish whether the effect on the enzyme is related to age and to site.

\section{METHODS}

Subjects. Intestinal tissue was obtained from 35 children who underwent: 1) revision or take-down of an ileostomy or colostomy, 2) resection of an intestinal stricture or obstruction, and 3) surgical cure of Hirschsprung's disease or intestinal atresia. We obtained 19 small intestinal specimens (four jejunal and 15 
ileal) and 16 colonic specimens. The ages ranged from 1 day to $16 \mathrm{yr}$ for patients from whom small intestine was obtained and from 6 months to $5 \mathrm{yr}$ for those from whom colon was obtained. The specimens used in this study were obtained from the edges of the resected specimens and light microscopy revealed normal morphology. Mild to moderate inflammation was occasionally present.

Three specimens came from the small intestine of 1-day-old patients. One specimen was resected for necrotizing enterocolitis and two for intestinal atresia. In all three specimens, the proximal margins were viable, and villous architecture was intact. In two specimens, a mild amount of mucosal congestion was present. In one of these, slight superficial inflammation was also present. As a group they were not histologically different from specimens obtained from older children.

This investigation was approved by the Institutional Review Board Committee on Human Investigation of Children's Hospital Medical Center, Cincinnati, $\mathrm{OH}$.

Preparation of homogenates from surgical intestinal specimens. Intestinal specimens were processed within $1 \mathrm{~h}$ from excision. Mucosa was scraped using a glass slide and scrapings were placed in $50 \mathrm{ml}$ of mannitol buffer ( $50 \mathrm{mM}$ mannitol, $2 \mathrm{mM}$ TRIS pH 7.1, 0.1 mM phenylmethyl sulfonil-fluoride) and homogenized in a Sorvall OMNI Mixer for $1 \mathrm{~min}$ at maximum speed. The homogenate was filtered through a nylon mesh to remove fat and fragments of connective tissue. Two volumes of sucroseEDTA-Dithiotreitol buffer $(25 \mathrm{mM}$ sucrose, $50 \mathrm{mM}$ TRIS pH 7.3, $1 \mathrm{mM}$ EDTA, $1 \mathrm{mM}$ Dithiotreitol) were added to the filtrate which was then centrifuged at $15,000 \times g$ for 20 min at $4^{\circ} \mathrm{C}$. The pellet was washed in $5 \mathrm{mM}$ TRIS pH 7.6 and resuspended to obtain a protein concentration of $10-40 \mu \mathrm{g} / 10 \mu \mathrm{l}$ and immediately tested for guanylate cyclase activity. Protein was measured by the method of Lowry et al. (18).

Preparation of soluble guanylate cyclase. The supernatant from the low speed centrifugation $(15,000 \times g)$ was collected and centrifuged at $105,000 \times g$ for $1 \mathrm{~h}$ at $4^{\circ} \mathrm{C}$. The resultant supernatant was concentrated approximately 25-fold using a Minicon concentrator (Millipore Corp., Danver, MA). Samples were immediately tested for guanylate cyclase activity. Recovery of protein was approximately $10 \%$ of the initial homogenate.

$E$. coli ST preparation. ST from E. coli strain $18 \mathrm{D}$ was purified by HPLC as previously described (19) and quantitated by amino acid analysis. The effect of ST on guanylate cyclase activity was tested over a concentration range of $2.5 \times 10^{-10} \mathrm{M}$ to $5 \times$ $10^{-6} \mathrm{M}$.

Guanylate cyclase assay. This was performed as soon as the preparation of the specimens was completed since storage of the homogenates at $-80^{\circ} \mathrm{C}$ resulted in a decrease of activity. The guanylate cyclase assay was performed by incubating the homogenate at $32^{\circ} \mathrm{C}$ in the presence of GTP and a GTP-regenerating mixture, with or without ST, as previously described $(16,20)$. Unless otherwise specified, incubation lasted $5 \mathrm{~min}$ and was started by adding intestinal protein to the reaction mixture which had been preincubated at $32^{\circ} \mathrm{C}$. Final volume was $100 \mu \mathrm{l}$. Formed cGMP was measured by an RIA as previously described (21).

Data presentation and analysis. All experimental points in the guanylate cyclase assay were performed in triplicate and cGMP formed was measured in duplicate. Guanylate cyclase is expressed as pmol cGMP formed $* \min ^{-1} * \mathrm{mg}$ protein ${ }^{-1}$. The effect of ST on guanylate cyclase activity is expressed as fold increase over basal level. A complete ST dose-response curve was performed on 20 specimens (nine small intestinal and 11 colonic specimens) since some specimens were insufficient for complete analysis.

Results of ST dose-response curves were analyzed using the computer program "Allfit" (22). Regression analysis was performed using the computer program "Curfit." Statistical evaluation was performed using two-way analysis of variance. Data are presented as mean \pm SEM.

\section{RESULTS}

Effect of protein concentration. Basal as well as ST-stimulated guanylate cyclase activity of both small intestinal and colonic specimens were linear within a broad range of protein concentration. Figure 1 shows a representative experiment demonstrating linearity of basal and ST-stimulated guanylate cyclase activity over the range of $0.005-0.070 \mathrm{mg} / \mathrm{sample}$. All subsequent experiments were performed using 0.01-0.04 mg of intestinal protein/ sample.

Effect of time. Figure 2 shows guanylate cyclase activity of both small intestinal and colonic membranes measured at various times of incubation. Both basal and ST-stimulated guanylate cyclase activity were linear over the entire time period examined (2-60 min).

Small intestine: effect of age. Figure 3 shows the basal level of guanylate cyclase activity in the small intestinal membranes $(n$ $=18$ ). The activity ranged from $0.865-11.55$ pmol cGMP * $\min ^{-1} *$ mg protein ${ }^{-1}$. Guanylate cyclase activity appeared highest in specimens from the youngest patients and more variable, but generally lower in specimens from the older patients. In fact, the highest basal levels of guanylate cyclase were found in three small intestinal specimens from children 1 day of age (10.31 \pm $0.66 \mathrm{pmol}$ cGMP $* \min ^{-1} * \mathrm{mg}$ protein $\left.{ }^{-1}\right)$. However, we wish to be cautious in our interpretation of this data because of the very limited number of specimens from older children $(n=4$, greater than 24 months of age).

ST-stimulated guanylate cyclase in all specimens in a doserelated manner. Figure 4 shows the dose response curve from a representative experiment. In the small intestinal specimen from a 6-month-old patient progressive increase in guanylate cyclase activity occurred with increasing doses of ST until a plateau of 13.5-fold stimulation was reached at approximately $10^{-6} \mathrm{M}$. We chose the maximal fold increase over basal level and the $\mathrm{ED}_{50}$, i.e. the concentration of ST at which half maximal stimulation was obtained, as the respective parameters of responsiveness and sensitivity of guanylate cyclase to ST. Neither maximum fold stimulation nor $\mathrm{ED}_{50}$ directly correlated with basal level of guanylate cyclase.

Figure 5 shows the maximum ST-stimulated activity of small intestinal guanylate cyclase at various ages. ST produced a maximum fold increase over basal level ranging from 2.70- to 43.26fold. In a pattern similar to that observed with basal guanylate

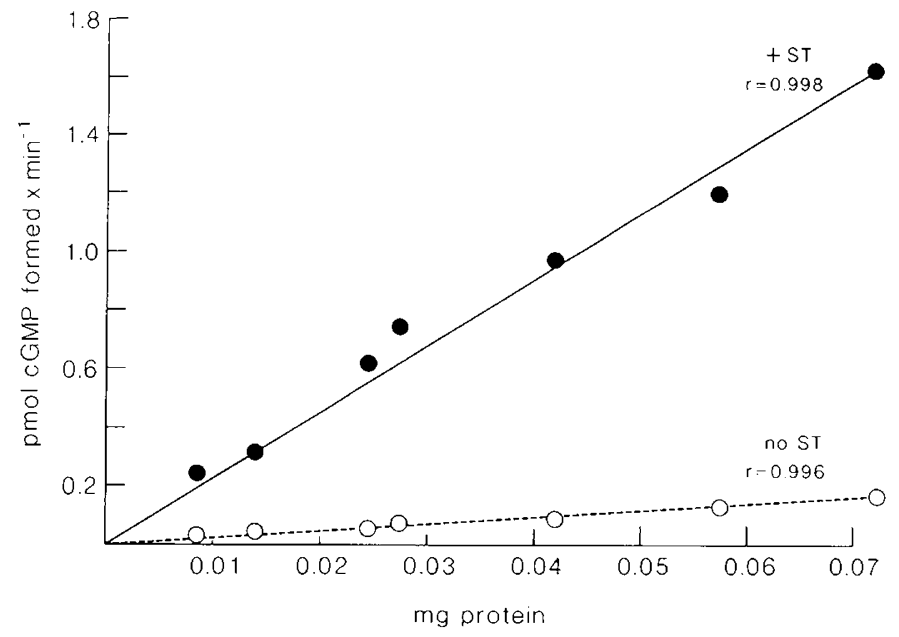

Fig. 1. Effect of protein concentration on basal and $E$. coli STstimulated guanylate cyclase activity. A representative experiment (ileum, 19 months) is shown. Increasing concentrations of intestinal protein were incubated with $(\bullet)$ and without $(O)$ ST $\left(5 \times 10^{-6} \mathrm{M}\right)$ for 5 $\min$ at $32^{\circ} \mathrm{C}$. Guanylate cyclase is measured as pmol cGMP $\times \min ^{-1} \times$ $\mathrm{mg}$ protein ${ }^{-1}$. Each point represents the average of three guanylate cyclase determinations. 


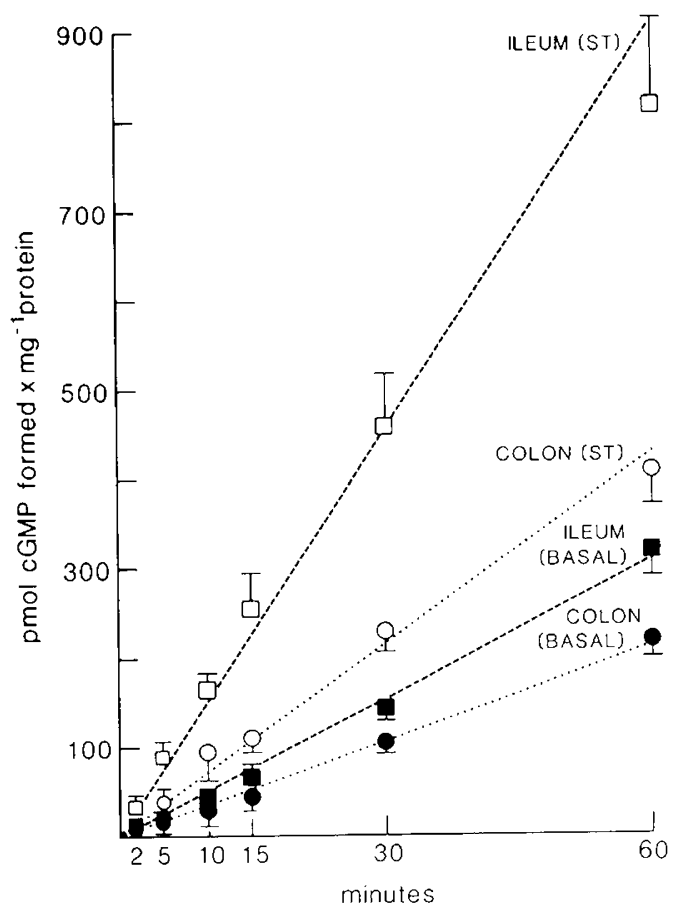

Fig. 2. Effect of time of incubation on basal and ST-stimulated guanylate cyclase activity. Intestinal membranes were incubated with and without ST $\left(5 \times 10^{-6} \mathrm{M}\right)$ for various periods of time at $32^{\circ} \mathrm{C}$. Values are means $\pm \mathrm{SE}$ of five different small intestinal specimens and four colonic specimens.

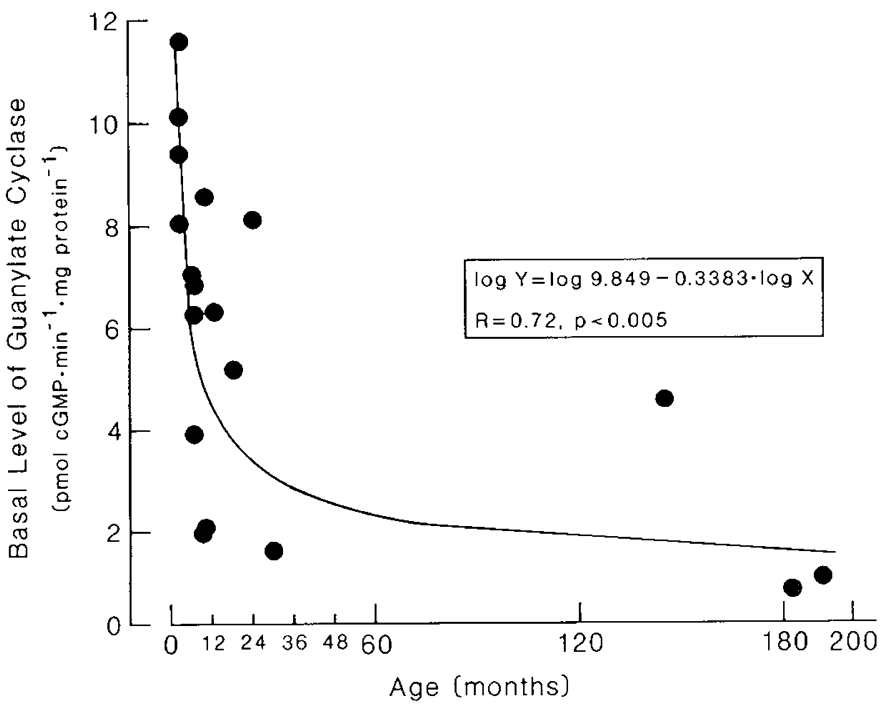

Fig. 3. Regression analysis of basal guanylate cyclase activity in small intestinal specimens from children aged 1 day to 192 months $(n=18)$. Each value represents the average of three determinations. Correlation coefficient was $0.72, p<0.005$.

cyclase activity (Fig. 3), ST-stimulated activity appeared highest in specimens from the youngest patients. The greatest stimulation induced by ST was observed in the small intestinal specimens from patients 1 day of age $(24.54 \pm 8.84$-fold increase over basal level). Again we wish to be cautious in the interpretation of these data because of the limited age distribution of the patients from whom specimens were available.

The $\mathrm{ED}_{50}$ of ST on small intestinal guanylate cyclase activity ranged from 0.84 to $8.80 \times 10^{-8} \mathrm{M}$ and did not correlate with the age of the patient from whom the specimen was obtained.

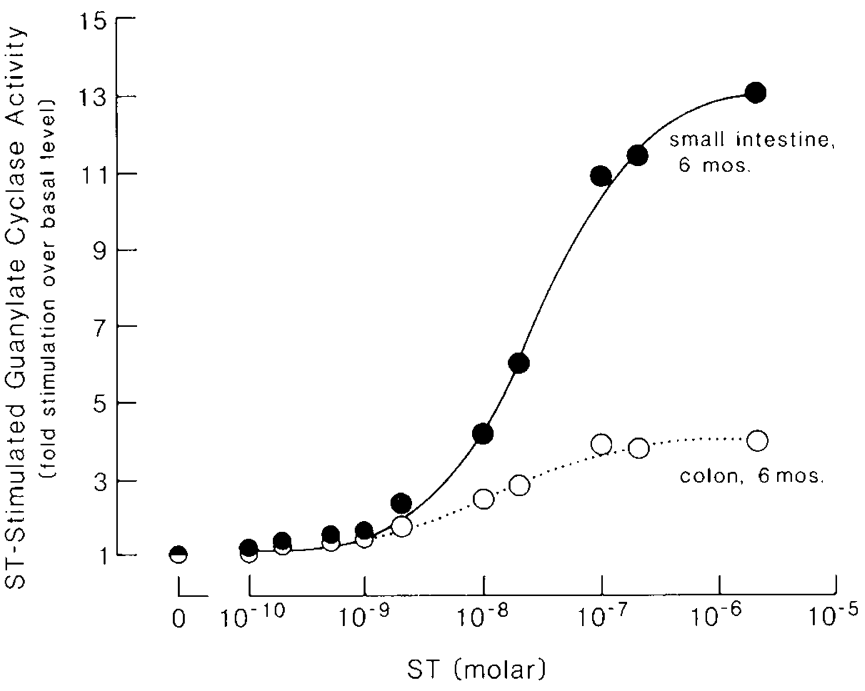

Fig. 4. Effect of various doses of ST on small intestinal (๑) and colonic $(O)$ guanylate cyclase activity in specimens from a 6 -month-old child. Guanylate cyclase activity is expressed as fold stimulation over basal level.

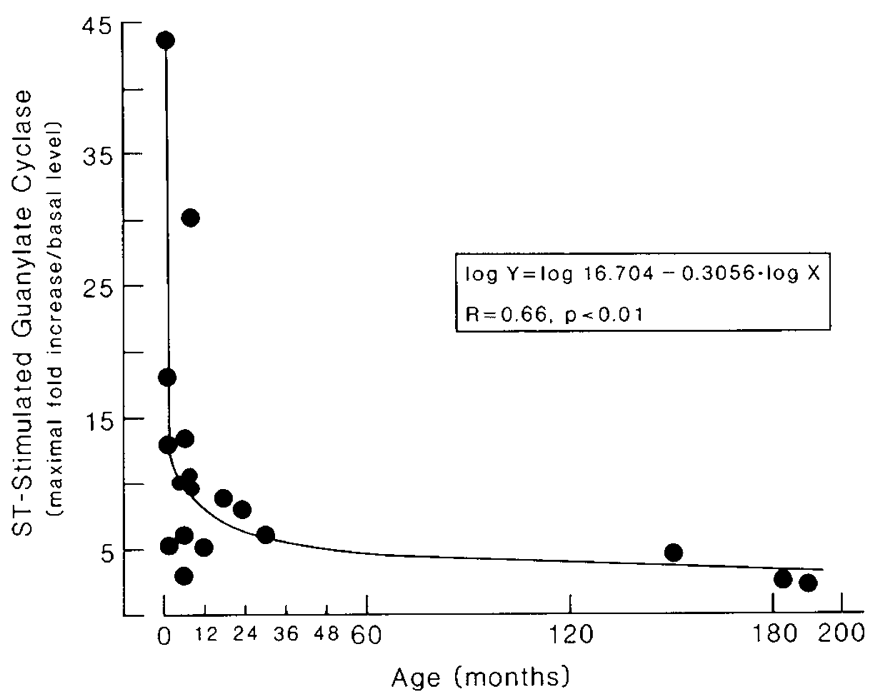

Fig. 5. Regression analysis of ST-stimulat'd small intestinal guanylate cyclase activity expressed as maximal fold increase over basal level, from children aged 1 day to 192 months $(n=18)$. Correlation coefficient was $0.66, \mathrm{p}<0.01$.

Colon: effect of age. In the colon, basal guanylate cyclase activity was variable over the range of age examined (6 month$48 / 12 \mathrm{yr}$ ). The basal levels varied between 0.86 to $7.81 \mathrm{pmol}$ cGMP $* \min ^{-1} * \mathrm{mg}$ protein ${ }^{-1}$. No colonic specimens were obtained from patients $<6$ month, an age range in which both the highest basal and ST-stimulated guanylate cyclase levels had been observed in the small intestinal specimens (Figs 3 and 5).

As was seen with the small intestinal membranes, ST-stimulated the activity of colonic guanylate cyclase in all specimens in a dose related manner. Increasing doses of ST, stimulated increased guanylate cyclase activity until a plateau was reached at approximately $10^{-6} \mathrm{M}$. A representative experiment is shown in Figure 4. In this colonic specimen from a 6-month-old patient maximal guanylate cyclase stimulation of 4 -fold over basal level was achieved.

In the 11 colonic specimens examined, ST produced a maximum fold increase over basal levels of colonic guanylate cyclase ranging from 1.45 to 8.30 -fold. This maximum fold increase 
over basal levels did not correlate with age over the age range examined.

The $\mathrm{ED}_{50}$ of $\mathrm{ST}$ on colonic guanylate cyclase was determined for the 11 specimens in which a full dose response curve was available. The $\mathrm{ED}_{50}$ ranged from 0.030 to $1.31 \times 10^{-8} \mathrm{M}$, but did not correlate with the age of the patient from whom the specimen was obtained.

For both the maximum fold stimulation over basal level and $\mathrm{ED}_{50}$ in the colon, our finding of a lack of correlation with age must be interpreted cautiously because of the somewhat limited age distribution of patients from whom specimens were obtained.

Small intestine versus colon. To evaluate the effects of site distinct from age, a two-way analysis of variance was performed on five paired (age-matched) specimens for basal guanylate cyclase activity, maximal ST-stimulated guanylate cyclase activity, and $\mathrm{ED}_{50}$ for ST-stimulated guanylate cyclase activity. The maximal ST-stimulated guanylate cyclase activity was significantly different $(p<0.001)$ between the small intestinal and colonic specimens. In general, the small intestinal stimulation was 2- to 3 -fold greater than in age-matched colonic specimens. This difference (13.5-versus 4-fold) is demonstrated in Figure 4 which shows dose-response curves for paired small intestinal and colonic specimens (age 6 months). There were no statistical differences in basal levels or $\mathrm{ED}_{50}$ for ST-stimulated guanylate cyclase activity.

Soluble guanylate cyclase. In the small intestine, soluble guanylate cyclase activity was $2.42 \pm 0.3$ pmol cGMP $* \min ^{-1} * \mathrm{mg}$ protein $^{-1}(n=3)$. Since the recovery of protein was approximately $10 \%$ of the initial homogenate, soluble guanylate cyclase activity represented a minimal fraction of total basal intestinal guanylate cyclase activity. One specimen was examined for soluble guanylate cyclase activity in the colon where the activity

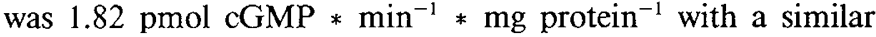
recovery of protein. ST had no stimulatory effect on soluble guanylate cyclase.

\section{DISCUSSION}

Basal as well as ST-stimulated guanylate cyclase activity were found in both the human small and large intestine. Both activities were linear with time and protein concentration and we therefore normalized our data by these parameters. De Rubertis and Craven (17), however, expressed cGMP content on the basis of tissue wet weight, DNA content, and protein concentration and found a lack of concordance among these methods. We did not measure weight or DNA content in our experiments. Therefore, we recognize that our data must be interpreted cautiously since we have used a single, albeit useful, method to normalize guanylate cyclase activity.

In the rat the main subcellular location of guanylate cyclase activity in the intestine is in the brush border (4). Since the brush border is substantially better developed in the small bowel compared to the colon, one might expect to find a greater guanylate cyclase activity in the small intestine than in the colon. However, in the human intestine we found a similar range of basal activity in the small intestine and in the colon. Furthermore, in paired, aged-matched specimens, including three specimens from the small intestine and colon of the same patient, basal guanylate cyclase activity was found to be similar in each site.

We did find that basal levels of small intestinal guanylate cyclase changed with age especially during the 1 st yr of life. Small intestinal guanylate cyclase activity from 1-day-old patients exceeded that of all other subjects. An age-related decrease of basal guanylate cyclase activity was observed with generally lower but more variable activity occurring after 12-24 months of age. The cause of such variability is uncertain, but could be related to the stimulatory effect of diet on guanylate cyclase as was proposed for cGMP (24), or other variables, e.g. medications.

The age distribution of subjects from whom colonic specimens were obtained and the observed variability of guanylate cyclase activity, did not permit us to establish if there was a pattern of basal guanylate cyclase activity related to age in the colon.

The stimulatory effect of ST on intestinal guanylate cyclase has been described in several animal models (5-8) and in a human derived colonic cell line (Guarino A, et al., unpublished data). ST-activation of guanylate cyclase is tissue specific $(6,23)$ and selectively involves particulate guanylate cyclase (23). In our study of the human small intestine, the effect of ST on guanylate cyclase was dose related and maximal stimulatory effect was related to age. The greatest stimulatory effect on guanylate cyclase was seen in specimens obtained from 1-day-old subjects (where the basal activity was also of the greatest magnitude). In general, ST induced a significantly greater stimulation of guanylate cyclase in the small intestine from younger children than older children. The number of specific receptors for ST in small intestine also appears to be greater in younger children (Cohen MB, et al., unpublished data). Therefore, ST binding to specific receptors and ST stimulation of guanylate cyclase followed a similar age-related pattern in the human small intestine, i.e. a larger number of receptors, capable of activating more guanylate cyclase, were present in younger children. These data might explain, at least in part, the increased severity of ST-induced diarrhea in younger children (25-27). In rats, such a correlation between ST binding and ST activation of guanylate cyclase has not been detected (16).

In contrast to the small intestine, in the colon there was no statistical relationship between the responsiveness of guanylate cyclase to stimulation by ST and age. This finding must be interpreted with the knowledge that we examined no colonic specimens from children under 6 months of age when the STinduced small intestinal stimulation was greatest.

In the rabbit, ST-activation of guanylate cyclase occurs all along the intestine with maximal effect in the duodenum and jejunum and a lesser effect in the distal colon (23). We found a similar pattern of regional responsiveness to ST in children with a significantly greater guanylate cyclase stimulation in the small intestine than in the colon. To discriminate between the effect of age and site on responsiveness of guanylate cyclase to ST, we examined ileal and colonic specimens obtained from five agematched specimens. ST resulted in a greater stimulation of guanylate cyclase activity in the ileum. In one other case, we examined jejunal and ileal specimens from the same patient and found that the effect of ST was significantly greater in the proximal segment. Finally, similar to findings described in animal models $(23,24)$, ST had no effect on soluble guanylate cyclase in humans.

In conclusion, in children 1) guanylate cyclase activity is present in both small and large intestine, largely in the particulate form. Basal activity is similar in both small intestine and colon. 2) In the small intestine, basal guanylate cyclase activity is related to age. Higher levels of basal activity were observed in younger patients. 3) Small intestinal guanylate cyclase is more responsive to ST than is colonic guanylate cyclase activity. Small intestinal guanylate cyclase stimulation by $E$. coli $\mathrm{ST}$ is greater in younger children than in older children.

Acknowledgments. The authors thank Dr. Kevin Bove and the Pathology Staff and the Surgical Staff of Children's Hospital Medical Center, Cincinnati, $\mathrm{OH}$ for their collaboration and Dr. Ralph Buncher, University of Cincinnati, for advice regarding statistical analysis.

\section{REFERENCES}

1. Murad F, Arnold WP, Mittal CK, Braughler JM 1979 Properties and regulation of guanylate cyclase and some proposed functions for cyclic GMP. Adv Cyclic Nucleotide Res 11:175-204

2. Ishikawa E, Ishikawa S, Davis JW, Sutherland E 1969 Determination of guanosine $3^{\prime}, 5^{\prime}$-monophosphate in tissues and of guanyl cyclase in rat intestine. J Biol Chem 244:6371-6376

3. Quill H, Weiser MW 1975 Adenylate and guanylate cyclase activity and cellular differentiation in rat small intestine. Gastroenterology 69:470-478

4. Walling MA, Mircheff AK, Van Os CH, Wright EM 1978 Subcellular distri- 
bution of nucleotide cyclases in rat intestinal epithelium. Am $\mathbf{J}$ Physiol 4:E539-E545

5. Field M, Graf LH, Laird WJ, Smith PL 1978 Heat-stable enterotoxin of Escherichia coli: in vitro effects on guanylate cyclase activation, cGMP concentration, and on ion transport in small intestine. Proc Natl Acad Sci USA 75:2800-2804

6. Guerrant RL, Hughes JM, Chang B, Robertson DC, Murad F 1980 Activation of intestinal guanylate cyclase by heat-stable enterotoxin of Escherichia coli: studies of tissue specificity, potential receptors and intermediates. $J$ Infect Dis 142:220-228

7. Dreyfus LA, Jaso-Friedmann L, Robertson DC 1984 Characterization of the mechanism of action of Escherichia coli heat-stable enterotoxin. Infect Immun 44:493-501

8. Waldman SA, Kuno T, Kamisaki Y, Chang LY, Gariepy J, O'Hanley P, Schoolnik G, Murad F 1986 Intestinal receptor for heat-stable enterotoxin of Escherichia coli is tightly coupled to a novel form of guanylate cyclase. Infect Immun 51:320-326

9. Aimoto S, Takao T, Shimonishi Y, Hara S, Takeda T, Takeda Y, Miwatani T 1982 Amino-acid sequence of a heat-stable enterotoxin produced by enterotoxigenic Escherichia coli. Eur J Biochem 129:257-263

10. Chan SK, Giannella RA 1981 Amino acid sequence of heat-stable enterotoxin produced by Escherichia coli pathogenic for man. J Biol Chem 255:77447746

11. Thompson MR, Luttrell M, Overmann G, Giannella RA 1985 Revised amino acid sequence for a heat-stable enterotoxin produced by an Escherichia col (strain 18D) pathogenic for man. Infect Immun 47:834-836

12. Giannella RA, Luttrell M, Thompson M 1983 Binding of Escherichia coli heat-stable enterotoxin to receptors on rat intestinal cells. Am J Physiol 245:G492-498

13. Guandalini S, Rao MC, Smith P, Field M 1982 cGMP modulation of ileal ion transport: in vitro effects of Escherichia coli heat stable enterotoxin. Am J Physiol 243:G36-G41

14. Guerrant RL, Moore RA, Kirshen PM, Sande MA 1975 Role of toxigenic and invasive bacteria in acute diarrhea of childhood. N Engl J Med 293:567-583

15. Black RE, Brown KH, Becker S, Yunus M 1982 Longitudinal studies and physical growth in rural Bangladesh: 1. Patterns of morbidity. Am J Epidemiol 115:305-314
16. Cohen MB, Moyer MS, Luttrell M, Giannella RA 1986 The immature rat small intestine exhibits an increased sensitivity and response to Escherichia coli heat-stable enterotoxin. Pediatr Res 20:555-560

17. De Rubertis F, Craven PA 1976 Calcium independent modulation of cyclic GMP and activation of guanylate cyclase by nitrosamines. Science 193:897899

18. Lowry OH, Rosebrough NJ, Farr AL, Randall RJ 1951 Protein measurement with the Folin phenol reagent. J Biol Chem 193:265-275

19. Thompson MR, Luttrell M, Overmann G, Giannella RA 1985 Biological and immunological characteristics of ${ }^{125} I-E$. coli heat-stable enterotoxin purified by HPLC. Anal Biochem 148:26-36

20. Waldman SA, O'Hanley PD, Falkow S, Schoolnik G, Murad F 1984 A simple sensitive and specific assay for heat-stable enterotoxin of Escherichia coli. J Infect Dis 149:83-89

21. Giannella RA, Drake KW 1979 Effect of purified $E$. col $i$ heat-stable enterotoxin on intestinal cyclic nucleotide metabolism and fluid secretion. Infect Immun 24:19-23

22. Teicher MH 1982 Applesoft dose-response analysis program written in Applesoft Basic. Med 40, Biomedical Computing Technology Information Center, Nashville, TN

23. Rao MC, Guandalini S, Smith P, Field M 1980 Mode of action of heat-stable Escherichia coli enterotoxin. Tissue and subcellular specificity and role of cyclic GMP. Biochim Biophys Acta 632:36-46

24. Hahn P Cannon de Rodriguez W Skala JP 1986 Effect of age and diet on cyclic nucleotide concentrations in the intestinal mucosa of developing rats. J Nutr 116:887-892

25. Black RE, Brown KH, Beckers S, Alim AR, Huq I 1982 Longitudinal studies of infectious diseases and physical growth in rural Bangladesh. II. Incidence of diarrhea and association of known pathogens. Am J Epidemiol 115:315324.

26. Black RE, Huq I, Merson MH, Alim ARMA, Yunus MD 1981 Incidence and severity of rotavirus and Escherichia coli diarrhea in rural Bangladesh. Implications for vaccine development. Lancet 1:141-143.

27. Guerrant RL, Kirchhoff LV, Shields DS, Nations LL, Sauer KT, McClelland KE, Trowbridge FL, Hughes JM 1983 Prospective study of diarrheal illness in northeastern Brazil: Patterns of disease, nutritional impact, etiologies and risk factors. J Infect Dis 148:986-997 